\title{
Evidence for the Contribution of NOSI Gene Polymorphism (rs3782206) to Prefrontal Function in Schizophrenia Patients and Healthy Controls
}

\author{
Zhifang Zhang ${ }^{1,2}$, Xiongying Chen ${ }^{1,2}$, Ping Yu ${ }^{1,2}$, Qiumei Zhang ${ }^{3}$, Xiaochen Sun ${ }^{1,2}$, Huang Gu ${ }^{1,2}$, \\ Hao Zhang ${ }^{4,5}$, Jinguo Zhai ${ }^{3}$, Min Chen ${ }^{3}$, Boqi Du, ${ }^{1,2}$, Xiaoxiang Deng ${ }^{1,2}$, Feng $\mathrm{Ji}^{3}$, Chuanyue Wang ${ }^{6}$, \\ Yutao Xiang ${ }^{6,7}$, Dawei $\mathrm{Li}^{8}$, Hongjie $\mathrm{Wu}^{9}$, Jun $\mathrm{Li}^{*}{ }^{, 1,2}$, Qi Dong ${ }^{1,2}$ and Chuansheng Chen ${ }^{10}$ \\ 'State Key Laboratory of Cognitive Neuroscience and Learning and IDG/McGovern Institute for Brain Research, Beijing Normal University, Beijing, \\ China; ${ }^{2}$ Center for Collaboration and Innovation in Brain and Learning Sciences, Beijing Normal University, Beijing, China; ${ }^{3}$ School of Mental \\ Health, Jining Medical University, Jining, Shandong Province, P.R. China; ${ }^{4}$ College of Precision Instrument and Optoelectronics Engineering, Tianjin \\ University, Tianjin, P. R. China; ${ }^{5}$ Key Laboratory of Opto-electronics Information Technology, Ministry of Education, Tianjin, China; ${ }^{6}$ Beijing Anding \\ Hospital, Beijing, China; ${ }^{7}$ Faculty of Health Sciences, Macau University of Science and Technology, Taipa, Macau, China; ${ }^{8}$ Center for Cognitive \\ Neuroscience, Duke University, Durham, NC, USA; ${ }^{9}$ Shengli Hospital of Shengli Petroleum Administration Bureau, Dongying, Shandong province, \\ P.R. China; ${ }^{10}$ Department of Psychology and Social Behavior, University of California, Imvine, CA, USA
}

\begin{abstract}
Nitric oxide (NO), a gaseous neurotransmitter, has been implicated in the pathogenesis of schizophrenia. Accordingly, several polymorphisms of the gene that codes for the main NO-producing enzyme, the nitric oxide synthase I (NOSI), have been found to convey a risk for schizophrenia. This study examined the role of NOSI gene polymorphisms in cognitive functions and related neural mechanism. First, with a sample of 580 schizophrenia patients and 720 healthy controls, we found that rs3782206 genotype had main effects on the I-back task $(P=0.005)$, the 2-back task $(P=0.049)$, the AY condition of the dot-pattern expectancy (DPX) task $(P=0.00 \mathrm{I})$, and the conflict effect of the attention network (ANT) test $(P<0.00 \mathrm{I}$ for RT differences and $P=0.002$ for RT ratio) and interaction effects with diagnosis on the BX condition of the DPX $(P=0.009)$, the AY condition of the DPX $(P<0.00 \mathrm{I})$, and the Stroop conflict effect $(P=0.003$ for RT differences and $P=0.038$ for $R T$ ratio). Simple effect analyses further showed that the schizophrenia risk allele $(T)$ of rs3782206 was associated with poorer performance in five measures for the patients ( 1 -back, $P=0.025 ; B X, P=0.017 ; A Y$, $P<0.00$ I; ANT conflict effect (RT differences), $P=0.005$; Stroop conflict effect (RT differences), $P=0.019$ ) and three measures for the controls ( for the 2-back task, $P=0.042$; for the ANT conlict effect (RT differences), $P=0.013$; for the ANT conflict effect (RT ratios), $P=0.028)$. Then, with a separate sample of 78 healthy controls, we examined the association between rs 3782206 and brain activation patterns during the $\mathrm{N}$-back task and the Stroop task. Whole brain analyses found that the risk allele carriers showed reduced activation at the right inferior frontal gyrus (IFG) during both tasks. Finally, we examined functional connectivity seeded from the right IFG to the dorsolateral prefrontal cortex (DLPFC) and anterior cingulate cortex under three conditions (the N-back task, the Stroop task, and the resting state). Results showed reduced connectivity with the DLPFC for the risk allele carriers mainly in the Stroop task and the resting state. Taken together, results of this study strongly suggested a link between NOSI gene polymorphism at rs3782206 and cognitive functions and their neural underpinnings at the IFG. These results have important implications for our understanding of the neural mechanism underlying the association between NOSI gene polymorphism and schizophrenia.

Neuropsychopharmacology (2015) 40, I383-1394; doi:I0.1038/npp.20।4.323; published online I8 February 2015
\end{abstract}

\section{INTRODUCTION}

Nitric oxide (NO) is a gaseous neurotransmitter that has been implicated in the pathogenesis of schizophrenia (SCZ)

*Correspondence: Dr J Li, State Key Laboratory of Cognitive Neuroscience and Learning and IDG/McGovern Institute for Brain Research, Beijing Normal University, 19\# Xinjiekouwai Road, Beijing 100875, China, Tel: +86 10 5880|755, Fax: +86 10 58801755, E-mail: lijundp@bnu.edu.cn

Received II August 2014; revised 2 December 2014; accepted 4 December 2014; accepted article preview online 10 December 2014
(Ramirez et al, 2004; Yao et al, 2004). This may be due to the fact that NO is a second messenger of the $N$-methylD-aspartate (NMDA) receptor (Snyder and Ferris, 2000) and that it interacts with both dopaminergic and serotonergic systems. Because $>90 \%$ of NO is catalyzed by nitric oxide synthase 1 (NOS1, also known as neuronal nitric oxide synthase, nNOS) (Brenman and Bredt, 1997), NOS1 was also thought to be involved in the pathogenesis of SCZ (Baba et al, 2004; Bernstein et al, 2005). Accordingly, NOS1 has become a candidate gene for SCZ. Reif et al (2006, 2009) found significant associations between two polymorphisms in the promoter region of the NOS1 gene (a VNTR and 
rs41279104) and SCZ. Two independent studies in Asia, each of which involved two independent samples and genotyped multiple polymorphisms to cover the NOS1 gene, did not replicate the results of Reif et al $(2006$; 2009) but found that another SNP in intron 3 (rs3782206) was associated with SCZ (Okumura et al, 2009; Tang et al, 2008). A genome-wide association study identified a third SNP (rs6490121) in intron 2 as one of the most significant SNPs that showed associations with SCZ (O’Donovan et al, 2008).

Further support of an association between NOS1 gene polymorphisms and SCZ came from studies of postmortem brain tissues, animal models, and brain MRI. First, postmortem studies on SCZ patients showed abnormalities of both NO and NOS1 in the prefrontal cortex (Akbarian et al, 1993; Xing et al, 2002). Second, animal studies on NOS1 gene knockout mice showed multiple cognitive dysfunctions related with the prefrontal cortex (Tanda et al, 2009; Zoubovsky et al, 2011). Recently, several imaging genetic studies have examined associations between the NOS1 gene polymorphisms and prefrontal cortex functions (mostly executive functions such as working memory and attentional control). Most of these studies were conducted by Reif et al $(2009 ; 2011)$ and focused on the promoter VNTR. For example, they found that the VNTR had a significant role in impulsive behavior (Retz et al, 2010; Laas et al, 2010; Reif et al, 2011) and mental disorders characterized by impulsive behavior (Reif et al, 2009). They also conducted a series of functional near-infrared spectroscopy (NIRS) studies and found that the VNTR could affect prefrontal activity during a working memory (a letter $\mathrm{N}$-back) task or an attentional control task (combining the stop-signal task with the Go/No-Go task) (Kopf et al, 2012; Kopf et al, 2011). Their electrophysiological studies using the continuous performance test (CPT) found similar results (Reif et al, 2006; Reif et al, 2009). Reif et al (2011) also conducted an fNIRS study on rs41279104 and found that it had a significant role in prefrontal cortex during a verbal fluency task (working memory). For another SNP, rs6490121, Donohoe et al (2009) found that it was significantly associated with working memory but not attentional control in two independent samples. The same group of researchers also conducted an fMRI study (Rose et al, 2012) with a spatial working memory task on 48 healthy controls and found that the risk allele carriers exhibited altered activation at the prefrontal cortex. Thus far, no studies have examined potential associations between rs3782206 and prefrontal functions.

In this study, we also focused on working memory and attentional control. We explored the association between NOS1 gene polymorphisms (rs3782206 and rs6490121) and these cognitive functions together with their related neural mechanism. We did not include polymorphisms in the promoter region (a VNTR and rs41279104), because their associations with SCZ were not replicated in the two studies of Asian population (Okumura et al, 2009; Tang et al, 2008). We conducted three analyses. First, we examined potential associations between the two SNPs and prefrontal functions (working memory as measured with the dot pattern expectation test (DPX) and the N-back task and attentional control as measured with the attention network test (ANT) and the Stroop task). Subjects included 580 SCZ patients and 720 healthy controls. We hypothesized that the SCZ risk alleles of the NOS1 gene would be associated with poor working memory and attentional control. Second, we conducted an fMRI study with a working memory task (the N-back task) and an attentional control task (the Stroop task) in 78 healthy volunteers. We hypothesized that the risk allele could affect activation at certain common brain regions subserving both working memory and attentional control. Finally, we investigated whether genotype affected the functional connectivities seeded from the inferior frontal gyrus (IFG) (based on the above analysis) to the dorsolateral prefrontal cortex (DLPFC) and anterior cingulate cortex (ACC). The choice of the DLPFC and ACC was based on recent meta-analyses of fMRI studies that have identified the DLPFC and ACC, in addition to the IFG, as commonly activated brain regions by different tasks of working memory and attentional control (Duncan and Owen, 2000; Minzenberg et al, 2009). In this analysis, we used both task fMRI data from the N-back and Stroop tasks and resting-state fMRI data, the latter of which reflects brain activity with no burden of a cognitive task.

\section{MATERIALS AND METHODS}

This study's protocol was reviewed and approved by the Institutional Review Board of the Institute of Cognitive Neuroscience and Learning at Beijing Normal University. All subjects were Han Chinese and gave written informed consent for this study.

\section{Study I: The Behavior Study}

Subjects. The sample used in this study has been described in published articles (Chen et al, 2012; Zhang et al, 2012; Zhu et al, 2013). Briefly, it consisted of 580 patients with SCZ and 720 healthy controls. The patients were recruited between August 2008 and December 2012 from the inpatients of the Ankang Hospital in Shandong Province. All patients fulfilled the ICD-10 criteria for SCZ according to the diagnostic consensus of two experienced psychiatrists. Patients were excluded if one of the psychiatrists was uncertain about their diagnosis. The positive and negative syndrome scale (PANSS) was used to assess each patient's positive (SAPS) and negative (SANS) symptoms. The mean score of the SAPS was $19.19 \pm 6.84$, the mean score of the SANS was $17.89 \pm 7.64$. All patients were treated with atypical antipsychotics. Exclusion criteria for the patients included a history of other psychiatric disorders and severe brain injury (any closed or open injuries that may be related to current symptoms or cognitive functions), current substance abuse, currently having acute psychotic episodes, and failure to cooperate during the cognitive tests. The healthy controls were from the same geographical region as the patients and were interviewed by experienced psychiatrists to screen for any personal or family history of psychiatric disorders. None of the patients and controls reported any history of hard drug use (eg, cocaine, crack, heroin, methamphatemine, etc.). Because older people are more likely to have certain chronic diseases such as long-term diabetes and hypertension that can affect their brain function, we limited our samples aged $<45$ years. 
Table I Demographic and Clinical Factors Across rs3782206 Genotypes by Diagnosis and for the Total Sample of Study I

\begin{tabular}{|c|c|c|c|c|c|}
\hline & \multicolumn{3}{|c|}{ Mean \pm SD } & \multirow[t]{2}{*}{ F or $\chi^{2}$} & \multirow[t]{2}{*}{$P$} \\
\hline & CC & CT & TT & & \\
\hline \multicolumn{6}{|l|}{ Age } \\
\hline Control & $25.75 \pm 8.44$ & $25.16 \pm 7.96$ & $26.85 \pm 8.95$ & 1.08 & 0.341 \\
\hline SCZ & $28.75 \pm 7.99$ & $27.88 \pm 7.56$ & $29.31 \pm 8.36$ & 1.36 & 0.257 \\
\hline Total & $27.17 \pm 8.36$ & $26.49 \pm 7.88$ & $28.14 \pm 8.70$ & 2.37 & 0.094 \\
\hline \multicolumn{6}{|c|}{ Gender (male/female) } \\
\hline Control & 182/204 & $126 / 157$ & $22 / 29$ & 0.66 & 0.717 \\
\hline SCZ & |98/II| & |46/80 & $34 / 11$ & 3.04 & 0.219 \\
\hline Total & $380 / 315$ & $272 / 237$ & $56 / 40$ & 0.81 & 0.668 \\
\hline \multicolumn{6}{|c|}{ Education (years) } \\
\hline Control & $9.40 \pm 3.44$ & $9.29 \pm 3.54$ & $8.96 \pm 4.15$ & 0.39 & 0.678 \\
\hline SCZ & $9.84 \pm 2.97$ & $9.94 \pm 2.99$ & $10.02 \pm 3.95$ & 0.14 & $0.87 \mid$ \\
\hline Total & $9.61 \pm 3.23$ & $9.61 \pm 3.29$ & $9.51 \pm 4.06$ & 0.04 & 0.957 \\
\hline \multicolumn{6}{|c|}{ PANSS positive } \\
\hline SCZ & $18.52 \pm 6.68$ & $|8.96 \pm 6.9|$ & $19.76 \pm 6.96$ & 0.99 & 0.374 \\
\hline \multicolumn{6}{|c|}{ PANSS negative } \\
\hline SCZ & $\mid 7.79 \pm 7.81$ & $17.66 \pm 7.76$ & $16.59 \pm 6.13$ & 0.62 & 0.536 \\
\hline \multicolumn{6}{|c|}{ Medication dose $(\mathrm{mg} / \text { day })^{\mathrm{a}}$} \\
\hline SCZ & $551.71 \pm 378.90$ & $618.32 \pm 450.08$ & $586.35 \pm 403.35$ & 2.16 & 0.116 \\
\hline
\end{tabular}

${ }^{\mathrm{a} C h l o r p r o m a z i n e ~ e q u i v a l e n t s . ~}$

Additional demographic information for both patients and the healthy controls is shown in Table 1.

The procedure for subject recruitment and test administration was the same for all subjects, including both patients and controls. A clinician first judged if the patient or the healthy control satisfied the inclusion and the exclusion criteria. Then the clinician and a psychologist together explained the study (including blood drawing and cognitive tests) and answered all questions the subject had. After the subject fully understood the study and signed informed consent document, the blood was drawn, and the subject was asked to perform the cognitive tests.

Cognitive tasks. All tasks have been introduced in our previous studies (Chen et al, 2012; Zhang et al, 2012; Zhu et al, 2013). Briefly, IQ was tested with Wechsler Adult Intelligence Scale-Revised, which was individually administered on paper and pencil. All the other tasks were administered using an IBM 14-inch screen notebook. The N-back task was a modified version of the task by Callicott et al (1998). Stimulus was a white circle presented randomly at one of the four corners of a grey diamond-shaped square. The four response buttons were also arranged in a diamond shape. Subjects were required to press one of the four buttons to match the target stimulus. Three task conditions (0-, 1- and 2-back) were used. Each condition (performed in one block) included 48 trials. All subjects followed the order of 0-, 1-, and 2-back conditions. Error rates of both 1- and 2-back trials were used as the main measures according to the twin study by Blokland et al (2008).

The DPX task was similar to that introduced by MacDonald et al (2005). It included 4 blocks, each of which included 40 trials (pairs of cues and probes). The stimuli were Braille font dot patterns. There were four conditions: AX, AY, BX, and BY, where A represented the valid cue, B the invalid cues (five in total), $\mathrm{X}$ the valid probe, and $\mathrm{Y}$ the invalid probes (five in total). Subjects were asked to press the target key when $\mathrm{A}$ was followed by $\mathrm{X}$ and press the non-target key in all other conditions. The 40 trials of each block consisted of 28 (70\%) AX trials, 5 (12.5\%) AY trials, 5 (12.5\%) BX trials, and 2 (5\%) BY trials. Error rate of the BX condition, error rate of the AY condition, and the difference between them (BX - AY) were used as the main measures.

A short version of the ANT (144 trials) was downloaded from Fan's webpage (http://www.sacklerinstitute.org/users/ jin.fan/). Stimuli were a central arrow and four flanker arrows (two for each side). Two conditions were involved: congruent condition (the flanker arrows were in the same direction as the central arrow) and incongruent condition (directions were opposite). Subjects were asked to respond according to the direction of the central arrow while ignoring flanker arrows (Fan et al, 2002). The conflict effect was calculated by subtracting the mean reaction time (RT) of the congruent condition from the mean RT of the incongruent condition. The conflict effect (RT differences) and its ratio (conflict effect/mean RT, which took into account the effects of overall mean RT) were used as the main measures.

A computerized version of the classic Stroop task was used in this study. Color words were presented in three different ink colors: red, green, and blue. The subjects were asked to press one of the three keys to indicate the color of ink while ignoring the meaning of the color word. The ink color and the word's meaning may be congruent or incongruent. The conflict effect and its ratio were calculated in the same way as described above for the ANT and were used as the main measures.

Genotyping. Genomic DNA was extracted using the standard method. Both SNPs, rs6490121 (A>G) and rs3782206 $(C>T)$, were genotyped using Taqman allele-specific assays on the 7900HT Fast Real-Time PCR System (Applied Biosystems, Foster City, CA, USA). The sample success rate for this SNP was $>95 \%$. The reproducibility of the genotyping was $100 \%$ according to a duplicate analysis of $10 \%$ of the genotypes.

Statistical analysis. The Hardy-Weinberg Equilibrium (HWE) test of the SNPs was done using the PLINK program (Purcell et al, 2007). All other analyses were done using SPSS version 17.0. Non-genetic factors, including age, gender, and years of education, across genotypes were compared by either one-way ANOVA or the chi-square test. The associations between cognitive function and SNPs were analyzed by two-way ANCOVA. In these analyses, genotype (for rs6490121, AA vs AG vs GG; for rs3782206, CC vs CT 
vs TT) and diagnosis (SCZ vs controls) were fixed factors. Following our previous studies, demographic factors, including age, gender, and years of education, were used as covariates. Significant main effects of genotype or significant interaction effects of genotype $\times$ diagnosis were followed up with the simple effects analysis of genotype in patients and controls separately. In the simple effects analysis, age, gender, and years of education were also used as covariates. Significance level was set at $P<0.025$ using Bonferroni's correction for the number of SNPs (2).

\section{Study II: The fMRI Study}

Subjects. A separate sample of 78 healthy controls was included in this study. Due to their excessive head motion $\left(>2 \mathrm{~mm}\right.$ or $2^{\circ}$ ), three subjects were excluded from the N-back fMRI analysis and two were excluded from the resting-state fMRI analysis. All subjects were recruited by advertisement and were interviewed by experienced psychiatrists to screen for any personal or family history of psychiatric disorders. All had normal or correctedto-normal vision and were right handed as assessed by the Edinburgh handedness inventory. Detailed demographic factors are shown in Table 3.

fMRI tasks. Because previous twin fMRI studies have demonstrated the usefulness of the N-back (Blokland et al, 2008) and the Stroop task (den Braber, 2012) in genetic studies, these two tasks were adapted for the current fMRI study. All subjects received training until their accuracy on both tasks showed no more improvement for several trials. The N-back task included two runs. Each run (lasting for $192 \mathrm{~s}$ ) consisted of eight blocks, in which the 2-back condition alternated with the 0-back condition in an A-B-BA manner (0-2-2-0). A centrally placed fixation cross was presented for $16 \mathrm{~s}$ before each set of four blocks of the task. Each block started with a 4-s on-screen instruction (either the number ' 0 ' or ' 2 ' on the center of screen indicating the type of working memory task to be performed). There were eight trials in each block. In each trial (lasting for $2 \mathrm{~s}$ ), the stimuli were presented for $500 \mathrm{~ms}$ followed by a 1.5-s blank. Subjects used a fiber-optic response box with four buttons arranged in a diamond shape. Subjects pressed one of the four buttons to match the target stimulus. The Stroop task consisted of 120 trials. Each trial began with a 500-ms fixation cross followed by the stimuli presented for $1 \mathrm{~s}$ and then a 2.5-s blank. The same response box as the N-back task was used. Subjects pressed the left button for red, the upper button for green, and the right button for blue. The bottom button was not used.

fMRI data acquisition. Imaging data were acquired at the Brain Imaging Center of Beijing Normal University. Subjects lay supine in a Siemens Trio 3T scanner with their head snugly fixed with straps and foam pads to restrict head movement. Resting-state images (240 volumes) were acquired first. Subjects were then moved out of the scanner and received training on the tasks. Back into the scanner, subjects were asked to perform the cognitive tasks while being scanned. The same echo-planar imaging sequence was used for both resting-state and task-related fMRI. Para- meters were as follows: repetition time $=2000 \mathrm{~ms}$; echo time $=30 \mathrm{~ms}$; flip angle $=90^{\circ}$; matrix size $=64 \times 64$; field of view $=200 \times 200 \mathrm{~mm}^{2} ; 31$ axial slices; $4.0 \mathrm{~mm}$ slice thickness without gap; and voxel size $=3.125 \times 3.125 \times 4.0 \mathrm{~mm}^{3}$.

Task fMRI data preprocessing and analysis. All image preprocesssing and analyses were implemented by using the statistical parametric mapping software (SPM8, Wellcome Department of Cognitive Neurology, London, UK). Preprocessing included slice timing (only for the fMRI data of the Stroop task), realignment, normalization to Montreal Neurological Institute (MNI) space, resampling to voxel size of $2 \times 2 \times 2 \mathrm{~mm}^{3}$, and smoothing with 8 - $\mathrm{mm}$ full-width at half maximum (FWHM) of the Gaussian smoothing kernel.

Contrast image for each subject was produced by firstlevel analysis: the 2-back condition minus the 0-back condition for the N-back task and the incongruent condition minus the congruent condition for the Stroop task. In these analyses, a high-pass filter at $128 \mathrm{~s}$ was used to remove noise associated with low-frequency confounds. The resulting images were then entered into a second-level analysis for the N-back task and the Stroop task separately. Two-sample $T$-test was used to test differences between the two genotypes (risk allele carriers $v s$ noncarriers) and subjects' age, gender, and years of education were controlled for. In the analysis of the N-back task, the accuracy of the 2-back task was also used as a covariate to exclude the effect of behavioral performance. Significance level was set at voxelwise $P<0.005$ and a cluster size of no fewer than 139 contiguous significant voxels, which would yield an Alphasim corrected threshold of $P<0.05$ as determined by the Alphasim program in the REST software (http://software.incf.org/ software/rest-a-toolkit-for-resting-state-fmri). To obtain the overlapping regions between these two tasks, we defined the significant clusters in the Stroop fMRI analysis as regions of interest (ROIs) by the MarsBar toolbox (http://marsbar. sourceforge.net/), then used these ROIs as masks and re-ran the second-level analysis of the N-back fMRI data.

We also conducted psychophysiological interactions (PPI) analyses (Friston et al, 1997) on task-related functional connectivity (FC) between the seed region (14 overlapping voxels between the two tasks) and the target regions of bilateral DLPFC and bilateral ACC. This analysis was also conducted using SPM8. Three regressors were used: the time series of the seed, the condition series of the two tasks, and the PPI term (ie, the interaction between the first two regressors). Two-sample $T$-test was used to compare PPI differences between genotypes controlling for age, gender, and years of education. Two ROIs were used: the bilateral DLPFC (BA9+BA46-inferior frontal gyrus) and the bilateral ACC (BA24 + BA32). Both were defined using the WFU PickAtlas software (http://fmri.wfubmc.edu/software/ PickAtlas) with a dilation factor of 2.0. Significance level was set at voxelwise $P<0.005$ and a cluster size of no fewer than 28 contiguous significant voxels in the ROI of bilateral DLPFC or no fewer than 40 in the ROI of bilateral ACC using the Alphasim program.

Resting-state fMRI data preprocessing and analysis. All image preprocessing was conducted using SPM8. In addition to slice timing, realignment, normalization (resampling 
to voxel size of $2 \times 2 \times 2 \mathrm{~mm}$ ), and smoothing ( $8 \mathrm{~mm}$ FWHM of the Gaussian smoothing kernel), images were detrended and bandpass filtered $(0.01-0.08 \mathrm{~Hz})$. All other analyses were conducted using the REST software (http:// software.incf.org/software/rest-a-toolkit-for-resting-state-fmri). After regressing out the effect of nuisance covariates that included the six head motion parameters as well as global mean signals and signals of both white matter and cerebrospinal fluid, mean time course of the seed was extracted, and the FC between the seed and the target regions were calculated. We then obtained an FC image for each subject and used two-sample T-test to compare FC differences between genotypes controlling for age, gender, and years of education. The seed and the targets as well as the associated significance threshold were the same as those described above for the PPI analyses.

\section{RESULTS}

\section{Study I: the Behavior Study}

Sample size varied by SNP genotype and cognitive task, because the DPX task and the Stroop task were added to data collection from patients later than the N-back task and the ANT and because of minor differences in the call rates of genotyping for the two SNPs. No deviation from HWE was found for either SNP (both $P$-values $>0.05)$. All cognitive measures showed normal or nearly normal distribution in both patients and controls (see Supplementary Table S1). All demographic and clinical data showed no significant differences across genotypes in patients, controls, and the total samples (all $P$-values $>0.05$ ) (see Table 1 and Supplementary Table S2).

For rs3782206, the main effect of genotype and/or the interaction effects of genotype $\times$ diagnosis were significant for eight of the nine measures. The only measure without a significant effect was $\mathrm{BX}-\mathrm{AY}\left(\mathrm{F}_{\text {genotype }}=0.53, P=0.588\right.$; $\left.\mathrm{F}_{\text {diagnosis } \times \text { genotype }}=0.28, P=0.760\right)$. Five measures showed a significant main effect of genotype, including the error rate of the 1-back task $\left(\mathrm{F}_{\text {genotype }}=5.33, P=0.005\right)$, the error rate of the 2-back task $\left(\mathrm{F}_{\text {genotype }}=3.03, P=0.049\right)$, the error rate for the AY condition of the DPX task $\left(\mathrm{F}_{\text {genotype }}=7.25\right.$, $P=0.001)$, and the conflict effect of the ANT $\left(\mathrm{F}_{\text {genotype }}=9.74, P<0.001\right)$ and its ratio $\left(\mathrm{F}_{\text {genotype }}=6.21\right.$, $P=0.002)$. Only the result for the error rate of the 2 -back test did not survive the Bonferroni's correction. Across these measures, the SCZ risk allele $(\mathrm{T})$ was associated with poor performance. When patients and controls were considered separately, the results were significant in patients for three measures (the 1-back task, $F=3.72$, $P=0.025$, partial $\eta^{2}=1.4 \%$; the error rate for the AY condition of the DPX task, $F=9.90, P<0.001$, partial $\eta^{2}=5.8 \%$; and the conflict effect of the ANT, $F=5.30$, $P=0.005$, partial $\left.\eta^{2}=1.9 \%\right)$. Tukey's post hoc analyses showed that the TT genotype performed worse than the other genotypes (the 1-back task, CC vs TT $P=0.151$, CT $v s$ TT $P=0.009$, CC $v s$ CT $P=0.092$; the error rate for the AY condition, CC vs TT $P=0.001$, CT vs TT $P<0.001$, CC vs CT $P=0.079$; and the conflict effect of the ANT, CC vs TT $P=0.028$, CT vs TT $P=0.001$, CC vs CT $P=0.149$ ). In healthy controls, significant results were found for the 2-back task $\left(F=3.18, P=0.042\right.$, partial $\left.\eta^{2}=1.0 \%\right)$ and the conflict effect of the ANT $(F=4.37, P=0.013$, partial $\left.\eta^{2}=1.3 \%\right)$ and its ratio $(F=3.60, P=0.028$, partial $\eta^{2}=1.1 \%$ ) (see Table 2). Tukey's post hoc analyses showed a similar pattern as in patients for the conflict effect of the ANT (CC vs TT $P=0.004$, CT vs TT $P=0.001, \mathrm{CC}$ vs CT $P=0.693$ ) and its ratio (CC vs TT $P=0.023$, CT vs TT $P=0.006$, CC vs CT $P=0.601$ ) (see Table 2).

The interaction effect of genotype $\times$ diagnosis was significant for four measures, including the error rate for both the $\mathrm{BX}\left(\mathrm{F}_{\text {diagnosis }} \times\right.$ genotype $\left.=4.72, P=0.009\right)$ and the AY condition of the DPX task $\left(F_{\text {diagnosis }} \times\right.$ genotype $=10.16$, $P<0.001)$ and the conflict effect of the Stroop task $\left(\mathrm{F}_{\text {diagnosis }} \times\right.$ genotype $\left.=5.90, \quad P=0.003\right)$ and its ratio $\left(\mathrm{F}_{\text {diagnosis }} \times\right.$ genotype $\left.=3.29, P=0.038\right)$. Only the result for the conflict effect's ratio of the Stroop task did not survive the Bonferroni's correction. Simple effects analyses showed that the interaction effects were driven by the significant genotype effect in the SCZ group (the error rate for the $\mathrm{BX}$ condition, $F=4.13, P=0.017$, partial $\eta^{2}=2.4 \%$; the error rate for the AY condition, $F=9.90, P<0.001$, partial $\eta^{2}=5.8 \%$; and the conflict effect of the Stroop task, $F=3.99, P=0$. 019, partial $\left.\eta^{2}=2.1 \%\right)$. Tukey's post hoc analyses also showed a similar pattern as above (for the error rate for the $\mathrm{BX}$ condition, CC $v s$ TT $P=0.219$, CT $v s$ TT $P=0.030$, CC $v s$ CT $P=0.187$; for the conflict effect of the Stroop task, CC vs TT $P=0.267$, CT vs TT $P=0.016$, CC vs CT $P=0.238)$. No simple effects were significant for the healthy controls (see Table 2). For rs6490121, none of the main effects of genotype or the interaction effects of genotype $\times$ diagnosis was significant (all $P$-values $>0.05$; see Supplementary Table S3). Therefore this SNP was not examined in the following fMRI study.

\section{Study II: The fMRI Study}

Due to the small sample size of the rs3782206 TT genotype (only six), we combined the TT genotype with the TC genotype to form the group of risk allele carriers. No significant difference was found between the carriers (TT/TC) and noncarriers (CC) in demographic factors (all $P$-values $>0.05$; see Table 3 ). Behavioral performances on both tasks were comparable between the two genotype groups (both $P$-values $>0.05$; see Table 3 ). Both tasks activated a widespread network as shown in Supplementary Figures S1 and S2.

For the N-back task, the whole-brain analysis (with $P$ set at 0.005 ) showed that risk allele carriers showed lower activation (stronger deactivation) at the right inferior frontal gyrus (IFG) (BA44) than the noncarriers (cluster size $=147$ voxels, $P_{\text {corrected }}=0.036$; peak voxel MNI coordinate: $\left.x=52, y=10, z=14, \mathrm{~T}=3.05, P_{\text {uncorrected }}=0.002\right)$. The carriers also showed lower activation than the noncarriers at the right inferior parietal lobule (IPL), although it did not survive the correction for multiple comparisons (BA40) (cluster size $=119$ voxels, $P_{\text {corrected }}=0.119$; peak voxel MNI coordinate: $x=64$, $y=-44, z=24, \mathrm{~T}=3.95, P_{\text {uncorrected }}<0.001$; see Figure 1 and Supplementary Figure S3).

Similarly, for the Stroop task, the whole-brain analysis (with $P$ set at 0.005 ) found that the carriers showed lower activation (stronger deactivation) than the noncarriers at 
Table 2 Cognitive Functions Across rs3782206 Genotypes

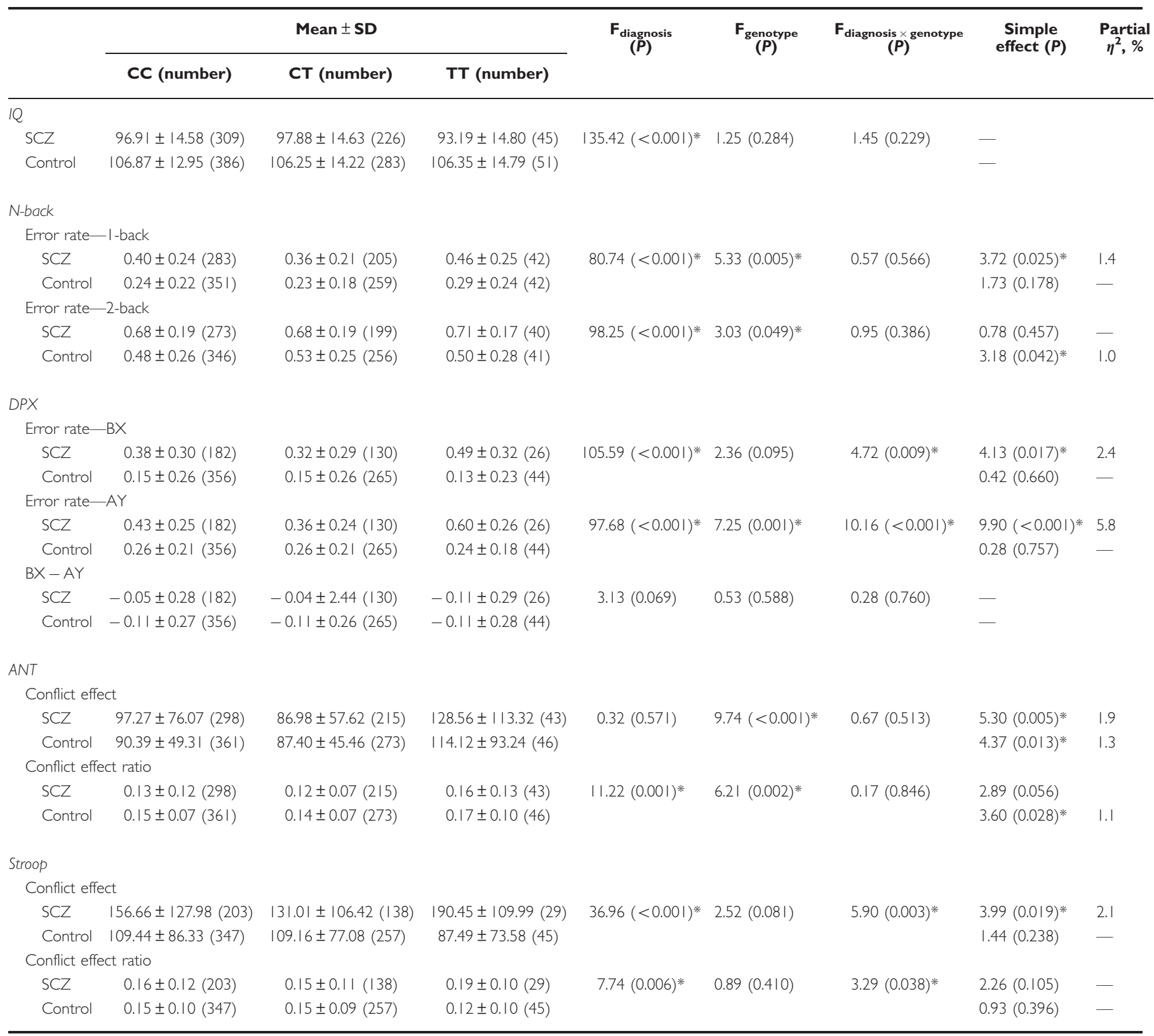

$* P<0.05$

the right IFG (BA44/45) (cluster size $=198$ voxels, $P_{\text {corrected }}=0.005$; peak voxel MNI coordinate: $x=58$, $y=14, z=18, \mathrm{~T}=3.40, P_{\text {uncorrected }}=0.001$; see Figure 2). This cluster is very near or even overlapping with the IFG cluster revealed in the N-back task (see Figure 1 and Supplementary Figure S4).

We then examined the FC between the seed region in the right IFG (the 14 overlapping voxels between the N-back task and the Stroop task) and bilateral DLPFC or bilateral ACC. For the Stroop task, compared with the noncarriers, the risk allele carriers showed a significantly decreased FC from the seed to bilateral DLPFC (for the left DLPFC, cluster size $=30$ voxels, $P_{\text {corrected }}=0.045$; peak voxel MNI coordinate: $x=-6, y=66, z=22, \mathrm{~T}=3.23, P_{\text {uncorrected }}=0.001$; and for the right DLPFC, cluster size $=90$ voxels, $P_{\text {correcte- }}$ ${ }_{d}<0.001$; peak voxel MNI coordinate: $x=20, y=64, z=28$, $\mathrm{T}=3.80, P_{\text {uncorrected }}<0.001$; see Figure 2$)$. For the $\mathrm{N}$-back task, only two voxels survived at $P<0.005$, with the peak at the right DLPFC (peak voxel MNI coordinate: $x=12, y=58$, $\left.z=22, \mathrm{~T}=2.75, P_{\text {uncorrected }}=0.004\right)$. No significant results were found at the bilateral ACC. When we set $P$ threshold at 0.05 and re-ran the analyses on the N-back task, we observed that, compared with the noncarriers, the carriers also showed decreased FC from the seed to bilateral DLPFC (for the left DLPFC, cluster size $=66$ voxels; peak voxel MNI coordinate: $x=-16, y=52, z=24, \mathrm{~T}=2.42, P_{\text {uncorrected }}=$ 0.009; and for the right DLPFC, cluster size $=60$ voxels; see Figure 2). Across tasks and significant clusters, the 
Table 3 Demographic Factors and Cognitive Task Performance Across rs3782206 Genotypes in Study II

\begin{tabular}{|c|c|c|c|c|}
\hline & \multicolumn{2}{|c|}{ Mean \pm SD } & \multirow[t]{2}{*}{ F or $\chi^{2}$} & \multirow[t]{2}{*}{$P$} \\
\hline & CC & CT/TT & & \\
\hline \multicolumn{5}{|l|}{ The N-back task } \\
\hline Gender (male/female) & $28 / 11$ & $28 / 8$ & 0.35 & 0.558 \\
\hline Age & $26.87 \pm 8.57$ & $27.72 \pm 5.97$ & 0.25 & 0.622 \\
\hline Accuracy-2-back & $0.87 \pm 0.13$ & $0.87 \pm 0.15$ & 0.01 & 0.938 \\
\hline RT-2-back & $363.29 \pm 118.07$ & $347.02 \pm 98.48$ & 0.42 & 0.521 \\
\hline \multicolumn{5}{|l|}{ The Stroop task } \\
\hline Gender (male/female) & $29 / 12$ & $28 / 9$ & 0.24 & 0.628 \\
\hline Stroop-conflict effect ratio & $0.24 \pm 0.12$ & $0.25 \pm 0.11$ & 0.07 & 0.790 \\
\hline Accuracy — congruent condition & $0.98 \pm 0.03$ & $0.99 \pm 0.02$ & 0.35 & 0.557 \\
\hline Accuracy_-incongruent condition & $0.90 \pm 0.14$ & $0.92 \pm 0.06$ & 0.88 & 0.352 \\
\hline \multicolumn{5}{|l|}{ Resting state } \\
\hline Gender (male/female) & $27 / 12$ & $28 / 9$ & 0.61 & 0.356 \\
\hline Age & $26.90 \pm 8.57$ & $27.68 \pm 5.89$ & 0.21 & 0.648 \\
\hline Education (years) & $13.54 \pm 3.04$ & $|3.03 \pm 3.0|$ & 0.54 & 0.463 \\
\hline IQ & | | $5.49 \pm 11.91$ & $117.17 \pm 9.73$ & 0.42 & 0.519 \\
\hline
\end{tabular}

noncarriers showed positive FC, whereas the carriers showed negative FC (see Supplementary Figure S5).

Finally, we analyzed the resting-state FC. At $P<0.005$, resting-state FC between the seed and DLPFC was also significantly reduced in the risk allele carriers as compared with the non-carriers but mainly in the left hemisphere (cluster size $=58$ voxels, $P_{\text {corrected }}=0.006$; peak voxel MNI coordinate: $x=-32, \quad y=26, \quad z=38, \quad \mathrm{~T}=3.30$, $\left.P_{\text {uncorrected }}=0.001\right)$. When the $P$ threshold was set at 0.05 , the risk allele carriers also showed reduced FC at the right DLPFC (cluster size $=237$ voxels; peak voxel MNI coordinate: $x=44, y=36, z=18, \mathrm{~T}=3.30, P_{\text {uncorrected }}=0.001$; see Figure 2 and Supplementary Figure S5).

\section{DISCUSSION}

Using data from a behavior study and an fMRI study, this article examined the associations between NOS1 gene polymorphisms and prefrontal cognitive functions and associated brain activities. The analysis of the behavioral data in Study I showed significant associations between rs3782206 of the NOS1 gene and performance on multiple cognitive tasks measuring both working memory (the DPX and the N-back task) and attentional control (the Stroop task and the ANT). The risk T allele was associated with worse performance. Functional MRI data from Study II showed that the risk $\mathrm{T}$ allele was associated with reduced activation at the right IFG and reduced FC between the right IFG and bilateral DLPFC. These results together indicated that the risk allele affected the function of the right IFG and its connection with DLPFC and consequently affected multiple cognitive functions.

Our results expanded the literature on the role of the NOS1 gene in cognitive functions and schizophrenia. First, we found significant associations between rs3782206 and working memory and attentional control, both of which have been associated with some symptoms of schizophrenia, such as distractibility, loosening of associations, disorganization, anger, aggression, and other socially inappropriate behaviors (Braver et al, 1999). In the previous animal studies, NOS1 gene knockout mice have showed very high levels of impulsive and aggressive behaviors (Chiavegatto et al, 2001; Chiavegatto and Nelson, 2003; Nelson et al, 2006). In the previous human studies, Reif et al (2009) found an association between a VNTR in the promoter region and many disorders characterized by impulsivity, such as attention deficit hyperactivity disorder, SCZ, and suicide attempt. They also found an association between the same VNTR and self-reported impulsiveness, venturesomeness, and empathy (Retz et al, 2010; Laas et al, 2010; Reif et al, 2011). They further found that the risk variant was associated with altered brain function related to working memory (in an fNIRS study with an N-back task) (Kopf et al, 2011) and attentional control (in both EEG and NIRS studies with the CPT and combined stop-signal and 

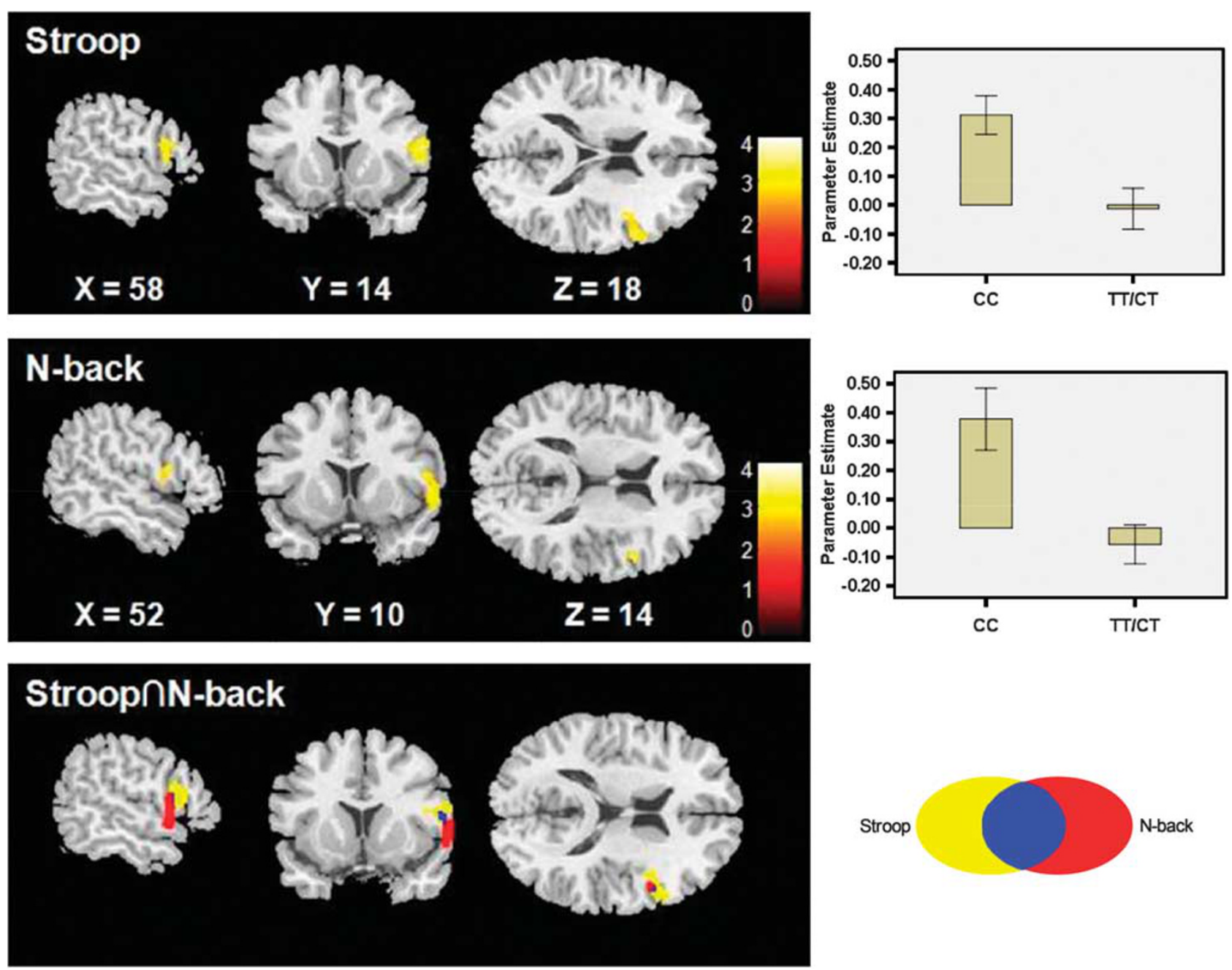

Figure I Whole brain comparisons between the CC and the TT/TC groups for the Stroop task and the N-back task. Compared with the CC group, the TT/TC (risk allele carrying) group consistently showed reduced activation at the right IFG $(P<0.005)$ with 14 voxels overlapping across the two tasks. The peak voxel MNI coordinates were as follows: for the Stroop task, $x, y, z=58,14$, I8; for the N-back task, $x, y, z=52,10,14$. The bar graph shows the mean activation of the significant clusters.

Go/No-Go task) (Kopf et al, 2012; Reif et al, 2006; Reif et al, 2009). Second, in this study, the risk allele of rs3782206 seemed to have a recessive effect on cognitive functions (the risk $\mathrm{T}$ allele homozygotes showed the worse performance than the other two genotypes). This pattern of a recessive effect was similar to previous findings for a different SNP, rs6490121, in Caucasians (Donohoe et al, 2009), in which the risk allele $(\mathrm{G})$ homozygotes performed worse than the other two genotypes. In contrast, for the VNTR, the risk allele seemed to have a dominant effect on cognitive functions, with the risk allele homozyogtes $(S / S)$ and the heterozygotes $(\mathrm{S} / \mathrm{L})$ showing worse performance than nonrisk allele homozygotes $(\mathrm{L} / \mathrm{L})$.

Our fMRI study (with the N-back and the Stroop tasks) consistently showed reduced activation at the right IFG for the risk allele carriers (the TT/TC group) (147 voxels at BA44 for the N-back task and 198 voxels at BA44/45 for the Stroop task). In other words, the main contribution of our study is that the NOS1 gene polymorphism seemed to affect cognitive functions via the right IFG. The IFG is one of the common brain regions for both working memory and attentional control (Aron et al, 2004, 2014). Common activations at the ventrolateral prefrontal cortex (BA47, the anterior part of the IFG) were found in a recent metaanalysis of $41 \mathrm{fMRI}$ studies on tasks that mainly included the N-back, AX-CPT, and Stroop tasks (Minzenberg et al, 2009). Another meta-analysis by Duncan and Owen (2000) found similar results. In terms of specific studies, Derrfuss et al (2004) conducted an fMRI study with three tasks (an N-back task, the Stroop task, and a task-switching paradigm) and found common activation at the inferior frontal junction (BA44/6/8, which is posterior to but overlapping with the IFG). The fMRI study by Fan et al (2003) of the ANT, the Stroop task, and a spatial conflict task also found overlapping activation at the IFG (BA44). Indeed, fNIRS studies by Kopf et al $(2011,2012)$ found that the risk allele homozygotes (S/S) of the NOS1 VNTR showed lower brain activity at the IFG as well as the DLPFC. The fNIRS study by Reif et al (2011) also found that the risk allele carriers (AA and AG) of rs41279014 showed reduced activation at lateral prefrontal cortex (mainly the IFG). 


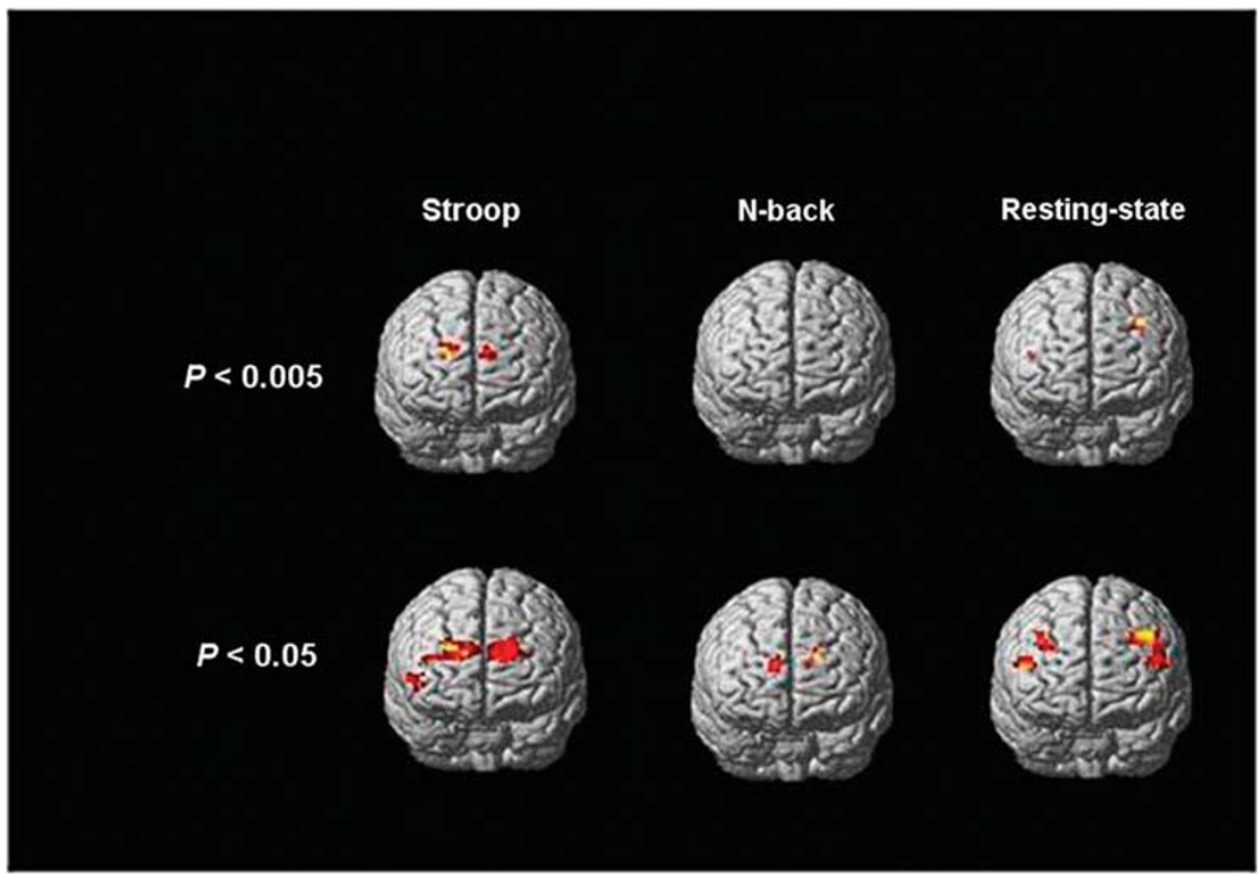

Figure 2 Functional connectivity between the right IFG and bilateral DLPFC was reduced in the risk allele carriers for all three conditions (the Stroop task, the N-back task, and the resting state). Results are shown at two significance levels $(P<0.005$ and $P<0.05)$.

Reduced activation at the IFG may suggest a dysfunction of this region. Broome et al (2009) compared brain activation in three groups (patients with the first episode of SCZ, individuals at risk for SCZ, and healthy controls) while they performed two working memory tasks (an N-back task and a verbal fluency task). They found significant group differences in activation at the IFG (BA44) with a consistent pattern of SCZ patients $<$ atrisks $<$ controls. A recent meta-analysis of $12 \mathrm{fMRI}$ studies of the N-back task also found reduced activation at the IFG in SCZ patients compared with healthy controls (Glahn et al, 2005). Our result suggested a link between the risk allele and a dysfunction of the right IFG. The right IFG, but not the left IFG, has been shown to be specifically important in executive functions. Patients with unilateral right IFG lesion showed impairment in a stop signal task; moreover, the greater the damage of the right IFG was, the worse the patients' performance was (Aron et al, 2003). Monkeys with lesion at the homologue of human right IFG also showed impairment in a Go/No-Go task (Goldman et al, 1970). Combined with our results at the behavioral level (Study I) that the risk allele was associated with worse working memory and attentional control, our fMRI results suggested a possible pathway from the risk allele of rs3782206 to dysfunction at the right IFG, and then to impairment in cognitive functions, and consequently to SCZ.

In addition to IFG, DLPFC and ACC also showed common activation across different tasks of executive functions in previous studies (Duncan and Owen (2000); Minzenberg et al, 2009). However, we did not find altered activation in these regions in the risk allele carriers. When we examined the FC between the right IFG and bilateral DLPFC or bilateral ACC, we consistently found that the risk allele carriers showed reduced FC between the right IFG and the bilateral DLPFC during the performance of both tasks as well as during resting state, although the result of the $\mathrm{N}$-back task did not survive the correction for multiple comparisons. One explanation is that the IFG and DLPFC have overlapping but also differentiated roles in cognitive functions. In addition to focusing attention on the target information and inhibiting irrelevant information, the IFG receives information from the posterior cortex and maintains it. The DLPFC, on the other hand, is responsible for higher order processing such as monitoring, comparison, and manipulation of information that is maintained in the IFG (Rowe et al, 2000; Wagner et al, 2001). Decreased FC between the IFG and DLPFC has been found in SCZ patients (Schlosser et al, 2003; Spence et al, 2000), which suggests a failure of functional integration between the IFG and DLPFC for SCZ patients. It needs to be mentioned that the risk allele carriers always showed negative FCs between the IFG and DLPFC during cognitive performance in this study, which may be because that the DLPFC was recruited when the IFG's activation was low (Egner, 2011; Petrides, 2000). As for the ACC, previous studies did not find any significant effects of the NOS1 gene. Specifically, for the VNTR and rs41279014, positive results were only found at the IFG and the DLPFC and not at the ACC (Kopf et al, 2012; Kopf et al, 2011). As for rs6490121, fMRI study by Rose et al (2012) only found significant results at the medial PFC, DLPFC, caudate, and cuneus but not at the ACC. Taken together these results, the ACC may not be an important brain region through which the NOS1 gene could affect cognitive functions.

In addition to the above major findings, two other findings of our study need to be discussed in light of inconsistent literature. First, significant relations between rs6490121 and IQ and working memory were found in a 
previous study by Donohoe et al (2009), but they were not replicated in our study even though the two studies had comparable sample sizes and both used well-validated tasks. Possible explanations for this inconsistency may include the different racial backgrounds of the samples and the different cognitive tasks used due to that many other studies have found racial differences in genetic effects (Wang et al, 2013) and that different working memory tasks have shown low inter-correlations (Conway et al, 2005). Future studies should include samples of different racial groups and use a broader range of measures to investigate whether the effect of NOS1 genetic variants is specific to some measures and samples.

Second, although we found significant results for the error rate of both $\mathrm{BX}$ and $\mathrm{AY}$ conditions, we did not find a significant result for the differential error rate of the $\mathrm{BX}-\mathrm{AY}$. The DPX task, a modified version of the expectancy AX-CPT task, is often used to assess the ability of context processing, an important aspect of working memory. It used pairs of cues ( $\mathrm{A}$ is a valid cue, all invalid cues were collectively referred to as $\mathrm{B}$ ) and probes (X or $\mathrm{Y}$ ) as stimuli. ' $\mathrm{X}$ ' is a valid probe only when it followed a valid cue ' $A$ ' (the AX condition). Errors under the BX condition indicated a failure of using the context information of the cue, whereas errors under the AY condition indicated that the effect of the cue was overly strong. Theoretically, the difference between the two conditions reflects the ability of context processing. However, some researchers have argued that, because most (70\%) of the trials of the DPX task are for the AX condition, there is an expectation that most Xs are valid probes and thus responses to the occasional (12.5\%) AY trials should be inhibited, just as for the No-Go condition of the Go/No-Go task. Therefore, in addition to context processing, the AY condition has a component of impulse inhibition, an important aspect of cognitive control, which complicates the interpretation of cognitive processes involved in the BX - AY scores.

Finally, some limitations of this study need to be mentioned. First, in addition to the SNPs included in this study, other polymorphisms of the NOS1 gene have been associated with SCZ and/or its endophenotypes in some of the previous studies (eg, the VNTR and the SNP at rs41279014) in Caucasian samples (Reif et al 2006, 2009), although not in Asian samples (Okumura et al, 2009; Tang et al, 2008). Future studies should include all risk polymorphisms in the NOS1 gene in order to provide a comprehensive understanding of this gene's role in SCZ. Second, our behavioral data showed a recessive effect of the risk allele of rs3782206 that the TT genotype performed worse than the other two genotypes, but due to the small number of subjects with the TT genotype in our fMRI data, we had to combine the TT and TC genotypes to form the group of $\mathrm{T}$ carriers. This strategy could have led to an underestimation of the recessive genetic effect. Future studies should use large sample sizes to specifically examine the recessive effect. Third, because we wanted to control for the potential confounds of premorbid intelligence level in this study of neural and genetic bases of SCZ and its cognitive endophenotypes, we tried to match the patients and the controls in terms of education, which could reflect premorbid IQ in patients. In addition, we used computerized tasks to measure cognitive functions in this study, which would be difficult for the patients who were seriously impaired or had a low level of education. However, there were still differences in IQ between patients and controls after controlling for their education level, age, and gender. Although such results were consistent with previous reports that patients should have lower IQ than controls (Donohoe et al, 2009), they nevertheless might have confounded to some extent our results between genetic variants and cognitive abilities because some of our cognitive measures were moderately correlated with IQ. In the end, one strength of the study (ie, the two groups matched in education) is also a weakness because the mean IQ of our SCZ sample was neither matched with that of the controls nor as low as typical SCZ patients. Finally, our fMRI version of the $\mathrm{N}$-back task used an A-B-B-A design, which may result in $B O L D$ response habituation and subjects' fatigue during the prolonged 2-back condition. Therefore, we need to be careful when comparing the results of this study to those of others that used different designs.

In conclusion, we found that the SCZ risk allele of rs3782206 was significantly associated with both working memory and attentional control. We also found that the risk allele was associated with significantly reduced activation at the right IFG and reduced FC between the right IFG and bilateral DLPFC. These results together furthered our understanding of the association between rs3782206 and SCZ and should be of value to theoretical models of the pathogenesis of SCZ that link genetic polymorphism, cognitive impairments, brain dysfunction, and SCZ.

\section{FUNDING AND DISCLOSURE}

This work was supported by grants from the National Key Basic Research Program of China (2014CB846103), the Beijing Higher Education Young Elite Teacher Project (YETP0249), the Open Research Fund of the State Key Laboratory of Cognitive Neuroscience and Learning (300072), the Natural Science Foundation of China (30800294), and the Fundamental Research Funds for the Central Universities. The authors declare no conflict of interest. The development project of medical science and technology in Shandong Province and Planning project of science and technology in Shandong Higher-education.

\section{REFERENCES}

Akbarian S, Bunney WE Jr., Potkin SG, Wigal SB, Hagman JO, Sandman CA et al (1993). Altered distribution of nicotinamideadenine dinucleotide phosphate-diaphorase cells in frontal lobe of schizophrenics implies disturbances of cortical development. Arch Gen Psychiatry 50: 169-177.

Aron AR, Fletcher PC, Bullmore ET, Sahakian BJ, Robbins TW (2003). Stop-signal inhibition disrupted by damage to right inferior frontal gyrus in humans. Nat Neurosci 6: 115-116.

Aron AR, Robbins TW, Poldrack RA (2004). Inhibition and the right inferior frontal cortex. Trends Cogn Sci 8: 170-177.

Aron AR, Robbins TW, Poldrack RA (2014). Inhibition and the right inferior frontal cortex: one decade on. Trends Cogn Sci 18: 177-185.

Baba H, Suzuki T, Arai H, Emson PC (2004). Expression of nNOS and soluble guanylate cyclase in schizophrenic brain. Neuroreport 15: 677-680. 
Bernstein HG, Bogerts B, Keilhoff G (2005). The many faces of nitric oxide in schizophrenia. A review. Schizophr Res 78: 69-86. Blokland GA, McMahon KL, Hoffman J, Zhu G, Meredith M, Martin NG et al (2008). Quantifying the heritability of taskrelated brain activation and performance during the N-back working memory task: a twin fMRI study. Biol Psychol 79: 70-79.

Braver TS, Barch DM, Cohen JD (1999). Cognition and control in schizophrenia: a computational model of dopamine and prefrontal function. Biol Psychiatry 46: 312-328.

Brenman JE, Bredt DS (1997). Synaptic signaling by nitric oxide. Curr Opin Neurobiol 7: 374-378.

Broome MR, Matthiasson P, Fusar-Poli P, Woolley JB, Johns LC, Tabraham P et al (2009). Neural correlates of executive function and working memory in the 'at-risk mental state'. Br J Psychiatry 194: $25-33$.

Callicott JH, Ramsey NF, Tallent K, Bertolino A, Knable MB, Coppola $\mathrm{R}$ et al (1998). Functional magnetic resonance imaging brain mapping in psychiatry: methodological issues illustrated in a study of working memory in schizophrenia. Neuropsychopharmacology 18: 186-196.

Chen M, Xu Z, Zhai J, Bao X, Zhang Q, Gu H et al (2012). Evidence of IQ-modulated association between ZNF804A gene polymorphism and cognitive function in schizophrenia patients. Neuropsychopharmacology 37: 1572-1578.

Chiavegatto S, Dawson VL, Mamounas LA, Koliatsos VE, Dawson TM, Nelson RJ (2001). Brain serotonin dysfunction accounts for aggression in male mice lacking neuronal nitric oxide synthase. Proc Natl Acad Sci USA 98: 1277-1281.

Chiavegatto S, Nelson RJ (2003). Interaction of nitric oxide and serotonin in aggressive behavior. Horm Behav 44: 233-241.

Conway ARA, Kane MJ, Bunting MF, Hambrick DZ, Wilhelm O, Engle RW (2005). Working memory span tasks: A methodological review and user's guide. Psychon B Rev 102: 769-786.

den Braber A, van 't Ent D, Cath DC, Veltman DJ, Boomsma DI, de Geus EJ (2012). Brain activation during response interference in twins discordant or concordant for obsessive compulsive symptoms. Twin Res Hum Genet 15: 372-383.

Derrfuss J, Brass M, von Cramon DY (2004). Cognitive control in the posterior frontolateral cortex: evidence from common activations in task coordination, interference control, and working memory. Neuroimage 23: 604-612.

Donohoe G, Walters J, Morris DW, Quinn EM, Judge R, Norton N et al (2009). Influence of NOS1 on verbal intelligence and working memory in both patients with schizophrenia and healthy control subjects. Arch Gen Psychiatry 66: 1045-1054.

Duncan J, Owen AM (2000). Common regions of the human frontal lobe recruited by diverse cognitive demands. Trends Neurosci 23: 475-483.

Egner T (2011). Right ventrolateral prefrontal cortex mediates individual differences in conflict-driven cognitive control. J Cogn Neurosci 23: 3903-3913.

Fan J, Flombaum JI, McCandliss BD, Thomas KM, Posner MI (2003). Cognitive and brain consequences of conflict. Neuroimage 18: 42-57.

Fan J, McCandliss BD, Sommer T, Raz A, Posner MI (2002). Testing the efficiency and independence of attentional networks. J Cogn Neurosci 14: 340-347.

Friston KJ, Buechel C, Fink GR, Morris J, Rolls E, Dolan RJ (1997). Psychophysiological and modulatory interactions in neuroimaging. Neuroimage 6: 218-229.

Glahn DC, Ragland JD, Abramoff A, Barrett J, Laird AR, Bearden CE et al (2005). Beyond hypofrontality: a quantitative meta-analysis of functional neuroimaging studies of working memory in schizophrenia. Hum Brain Mapp 25: 60-69.

Goldman PS, Rosvold HE, Mishkin M (1970). Evidence for behavioral impairment following prefrontal lobectomy in the infant monkey. J Comp Physiol Psychol 70: 454-463.
Kopf J, Schecklmann M, Hahn T, Dieler AC, Herrmann MJ, Fallgatter AJ et al (2012). NOS1 exlf-VNTR polymorphism affects prefrontal oxygenation during response inhibition tasks. Hum Brain Mapp 33: 2561-2571.

Kopf J, Schecklmann M, Hahn T, Dresler T, Dieler AC, Herrmann MJ et al (2011). NOS1 exlf-VNTR polymorphism influences prefrontal brain oxygenation during a working memory task. Neuroimage 57: 1617-1623.

Laas K, Reif A, Herterich S, Eensoo D, Lesch KP, Harro J (2010). The effect of a functional NOS1 promoter polymorphism on impulsivity is moderated by platelet MAO activity. Psychopharmacology 209: 255-261.

MacDonald AW 3rd, Goghari VM, Hicks BM, Flory JD, Carter CS, Manuck SB (2005). A convergent-divergent approach to context processing, general intellectual functioning, and the genetic liability to schizophrenia. Neuropsychology 19: 814-821.

Minzenberg MJ, Laird AR, Thelen S, Carter CS, Glahn DC (2009). Meta-analysis of 41 functional neuroimaging studies of executive function in schizophrenia. Arch Gen Psychiatry 66: 811-822.

Nelson RJ, Trainor BC, Chiavegatto S, Demas GE (2006). Pleiotropic contributions of nitric oxide to aggressive behavior. Neurosci Biobehav Rev 30: 346-355.

O'Donovan MC, Craddock N, Norton N, Williams H, Peirce T, Moskvina V et al (2008). Identification of loci associated with schizophrenia by genome-wide association and follow-up. Nat Genet 40: 1053-1055.

Okumura T, Okochi T, Kishi T, Ikeda M, Kitajima T, Yamanouchi Y et al (2009). No association between polymorphisms of neuronal oxide synthase 1 gene (NOS1) and schizophrenia in a Japanese population. Neuromolecular Med 11: 123-127.

Petrides M (2000). Dissociable roles of mid-dorsolateral prefrontal and anterior inferotemporal cortex in visual working memory. J Neurosci 20: 7496-7503.

Purcell S, Neale B, Todd-Brown K, Thomas L, Ferreira MA, Bender D et al (2007). PLINK: a tool set for whole-genome association and population-based linkage analyses. Am J Hum Genet 81: 559-575.

Ramirez J, Garnica R, Boll MC, Montes S, Rios C (2004). Low concentration of nitrite and nitrate in the cerebrospinal fluid from schizophrenic patients: a pilot study. Schizophr Res 68: 357-361.

Reif A, Herterich S, Strobel A, Ehlis AC, Saur D, Jacob CP et al (2006). A neuronal nitric oxide synthase (NOS-I) haplotype associated with schizophrenia modifies prefrontal cortex function. Mol Psychiatry 11: 286-300.

Reif A, Jacob CP, Rujescu D, Herterich S, Lang S, Gutknecht L et al (2009). Influence of functional variant of neuronal nitric oxide synthase on impulsive behaviors in humans. Arch Gen Psychiatry 66: 41-50.

Reif A, Kiive E, Kurrikoff T, Paaver M, Herterich S, Konstabel K et al (2011). A functional NOS1 promoter polymorphism interacts with adverse environment on functional and dysfunctional impulsivity. Psychopharmacology 214: 239-248.

Retz W, Reif A, Freitag CM, Retz-Junginger P, Rosler M (2010). Association of a functional variant of neuronal nitric oxide synthase gene with self-reported impulsiveness, venturesomeness and empathy in male offenders. J Neural Transm 117: 321-324

Rose EJ, Greene C, Kelly S, Morris DW, Robertson IH, Fahey C et al (2012). The NOS1 variant rs6490121 is associated with variation in prefrontal function and grey matter density in healthy individuals. Neuroimage 60: 614-622.

Rowe JB, Toni I, Josephs O, Frackowiak RS, Passingham RE (2000). The prefrontal cortex: response selection or maintenance within working memory? Science 288: 1656-1660.

Schlosser R, Gesierich T, Kaufmann B, Vucurevic G, Stoeter P (2003). Altered effective connectivity in drug free schizophrenic patients. Neuroreport 14: 2233-2237. 
Snyder SH, Ferris CD (2000). Novel neurotransmitters and their neuropsychiatric relevance. Am J Psychiatry 157: 1738-1751.

Spence SA, Liddle PF, Stefan MD, Hellewell JS, Sharma T, Friston KJ et al (2000). Functional anatomy of verbal fluency in people with schizophrenia and those at genetic risk. Focal dysfunction and distributed disconnectivity reappraised. $\mathrm{Br} J$ Psychiatry 176: 52-60.

Tanda K, Nishi A, Matsuo N, Nakanishi K, Yamasaki N, Sugimoto $\mathrm{T}$ et al (2009). Abnormal social behavior, hyperactivity, impaired remote spatial memory, and increased D1-mediated dopaminergic signaling in neuronal nitric oxide synthase knockout mice. Mol Brain 2: 19.

Tang W, Huang K, Tang R, Zhou G, Fang C, Zhang J et al (2008). Evidence for association between the 5' flank of the NOS1 gene and schizophrenia in the Chinese population. Int J Neuropsychopharmacol 11: 1063-1071.

Wagner AD, Maril A, Bjork RA, Schacter DL (2001). Prefrontal contributions to executive control: fMRI evidence for functional distinctions within lateral prefrontal cortex. Neuroimage 14: 1337-1347.

Wang Y, Li J, Chen C, Zhu B, Moysis RK, Lei X et al (2013). COMT rs4680 Met is not always the 'smart allele': Val allele is associated with better working memory and larger hippocampal volume in healthy Chinese. Genes Brain Behav 12: 323-329.

Xing G, Chavko M, Zhang LX, Yang S, Post RM (2002). Decreased calcium-dependent constitutive nitric oxide synthase (cNOS) activity in prefrontal cortex in schizophrenia and depression. Schizophr Res 58: 21-30.

Yao JK, Leonard S, Reddy RD (2004). Increased nitric oxide radicals in postmortem brain from patients with schizophrenia. Schizophr Bull 30: 923-934.

Zhang Q, Shen Q, Xu Z, Chen M, Cheng L, Zhai J et al (2012). The effects of CACNA1C gene polymorphism on spatial working memory in both healthy controls and patients with schizophrenia or bipolar disorder. Neuropsychopharmacology 37: 677-684.

Zhu X, Gu H, Liu Z, Xu Z, Chen X, Sun X et al (2013). Associations between TCF4 gene polymorphism and cognitive functions in schizophrenia patients and healthy controls. Neuropsychopharmacology 38: 683-689.

Zoubovsky SP, Pogorelov VM, Taniguchi Y, Kim SH, Yoon P, Nwulia E et al (2011). Working memory deficits in neuronal nitric oxide synthase knockout mice: potential impairments in prefrontal cortex mediated cognitive function. Biochem Biophys Res Commun 408: 707-712.

Supplementary Information accompanies the paper on the Neuropsychopharmacology website (http://www.nature.com/npp) 